\title{
Gesundheitskompetenz der Patienten stärken
}

\author{
Dieser Ausgabe von MMW liegt wieder „info praxisteam“ bei, das Magazin für Medizinische \\ Fachangestellte. Hauptthema in Heft 2/16 ist die Gesundheitskompetenz der Patienten. Um \\ diese zu stärken, bietet die AOK seit neuestem wissenschaftlich fundierte „Faktenboxen“ an.
}

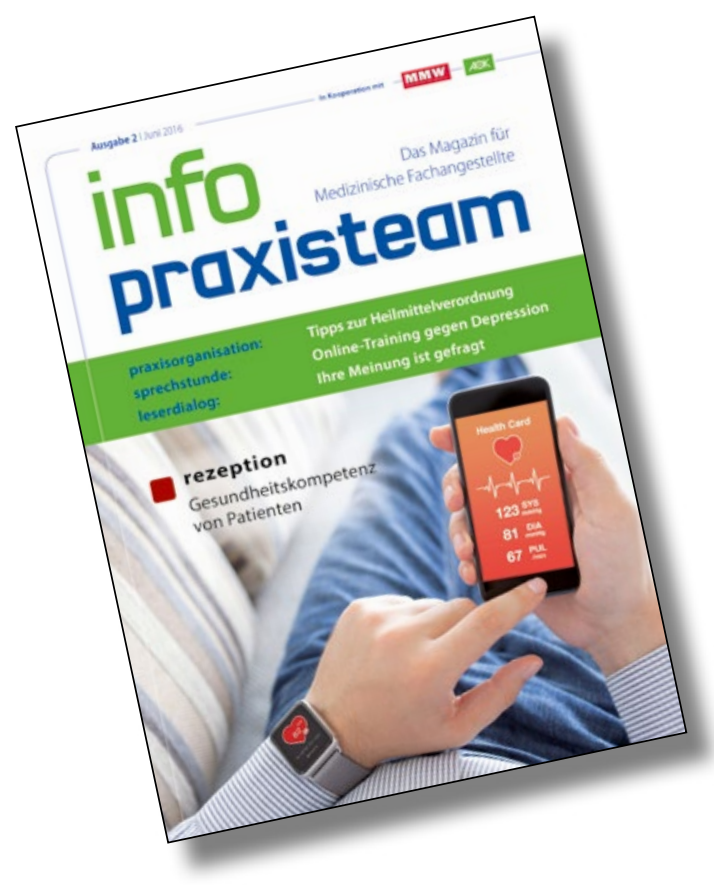

— Jeder Mensch muss fast täglich ganz unterschiedliche Entscheidungen für die Gesundheit treffen. Hier muss eine Information zu einem Krankheitsbild verstanden werden, dort geht es um die Entscheidung für oder gegen eine Impfung. Und bei jeder wirklichen gesundheitlichen Einschränkung stellt sich die Frage, ob ein Arztbesuch erforderlich ist oder nicht. ,info praxisteam “ beschäftigt sich daher ausführlich mit dem Thema Gesundheitskompetenz.

Wer mit seiner Gesundheit verantwortungsvoll umgehen soll, muss nicht nur die richtigen Informationen finden. Er muss sie auch verstehen, beurteilen und für sich anwenden können. Viele internationale Studien weisen darauf hin, dass eine mangelnde Gesundheitskompetenz negative gesundheitliche und finanzielle Auswirkungen hat:

- risikoreiches Gesundheitsverhalten,

- geringere Inanspruchnahme von Prävention und Früherkennung,
- spätere Diagnosestellung,

- geringere Therapietreue,

- schlechtere Fähigkeiten im Selbstmanagement,

- erhöhtes Risiko einer Krankenhausaufnahme,

- Risiko eines frühzeitigen Todes,

- allgemein schlechtere physische und psychische Gesundheit wie auch

- höhere Behandlungs- und Gesundheitskosten.

Neugierige, wertschätzende und akzeptierende Haltung

Das Wissenschaftliche Institut der AOK (WIdO) hat jetzt die erste bundesweite, repräsentative Untersuchung zur Gesundheitskompetenz in Deutschland vorgelegt. Die Ergebnisse zeigen, dass die Deutschen zu wenig über das Thema Gesundheit wissen. Die Gesundheitskompetenz unterscheidet sich dabei deutlich nach Alter, Geschlecht, Bildung und Einkommen.

Deshalb hat der AOK-Bundesverband gemeinsam mit Wissenschaftlern die „AOK-Faktenboxen“ entwickelt. Sie eignen sich auch als Unterstützung für das Patientengespräch. Die Boxen haben mehrere Teile: Auf Fragen rund um Nutzen und Risiken von Behandlungen, Untersuchungen, Früherkennungstests und Arzneimitteln folgt die Bewertung des Nutzens und des Schadens bzw. der möglichen Nebenwirkungen. Die Ergebnisse sind mit Grafiken und leicht verständlichen Texten aufbereitet.

Die Themen der Faktenboxen reichen von Impfungen (Masern/Mumps/Röteln, Influenza für Senioren, Pertussis für Jugendliche und Erwachsene) über Sinn und Unsinn von Nahrungsergänzungsmitteln, Röntgen bei allgemeinen Rückenbeschwerden, Stoßwellentherapie beim Tennisarm bis hin zum Nutzen eines jährlichen Ultraschalls zur Früherkennung von Eierstockkrebs.

Wer die Faktenboxen im Alltag nutzt, kann sicher sein, auf dem neuesten wissenschaftlichen Stand zu sein. Ganz bewusst sprechen die Wissenschaftler in den Boxen allerdings keine Empfehlungen aus. Der Leser soll lernen, Nutzen und Risiken selbst abzuwägen und auf dieser Grundlage eine Entscheidung für sich persönlich zu treffen - oder wenigstens gut informiert ins Gespräch mit dem Arzt gehen.

In der Rubrik „sprechstunde“ gibt es zwei Hauptbeiträge. Sie behandeln das Online-Training MoodGym, das Patienten mit depressiven Verstimmungen unterstützt, sowie die verschiedenen Ursachen von Schwindel. In der Rubrik „praxisorganisation“ geht es um die Verordnung von Heilmitteln.

Dr. Reinhard Merz

\section{„info praxisteam“ im Web}

Unser MFA-Magazin erscheint viermal jährlich. Alle Beiträge sind unter

www.info-praxisteam.de

auch im Internet abrufbar und können direkt kommentiert werden.

Auch für mobile Endgeräte wie Smartphones oder Tablets ist „info praxisteam“ verfügbar. Die App wird sowohl für iOS von Apple als auch für Geräte mit AndroidPlattform angeboten. Mehr darüber, wie man die App beziehen kann, erfahren Sie auf der oben angegebenen Website.

Zu den Beiträgen im Heft gibt es von der Redaktion ausgesuchte Verweise auf weiterführende Informationen online. Viele davon sind auch für Sie als Arzt interessant.

Sie erreichen uns per E-Mail unter:

redaktion@info-praxisteam.de 